\title{
An Electronic Medical Record system for Sri Lankan general practitioners
}

\author{
Dr. M.H.B. Ariyaratne \\ Postgraduate Institute of Medicine, University of Colombo, Sri Lanka. \\ Dr. G. Dissanayake \\ Postgraduate Institute of Medicine, University of Colombo, Sri Lanka. \\ Dr. C. Widisinghe \\ Postgraduate Institute of Medicine, University of Colombo, Sri Lanka. \\ Dr. J. Samarasekara \\ Postgraduate Institute of Medicine, University of Colombo, Sri Lanka.

\section{Dr. V. Banduwardhana} \\ Postgraduate Institute of Medicine, University of Colombo, Sri Lanka. \\ Contact e-mail address: buddhika.ari@gmail.com \\ eHealth Sri Lanka 2010,1(suppl.1):S38 \\ DOI: http://dx.doi.org/10.4038/sljbmi.v1i0.3691
}

Only the Abstract is available

\begin{abstract}
Usage of computer software in general medical practice is becoming a routine not only in developed countries like UK, but also in developing countries like India. Yet the popularity of software in Sri Lankan general practice is very low due to several reasons of which some are identified and addressed in developing a "Sri Lankan" Electronic Medical Record system.

The system was developed by five Sri Lankan doctors to address the issues they face in their own practice. During the system analysis, several other general practitioners have been consulted and they discussed the requirements, limitations, and other issues in detail.

One issue is the lack of time available to spend on the computer system. To make matters worse, most do not have that competency to handle the computers quick enough. What we finally achieved is a system which needs minimal doctor-computer interaction which can generate prescriptions faster than writing it by hand. This was achieved by features we call Diagnosis fills, Medicines fills, Patient refills, Visit refills, Right click alternatives for medicines and doses, Patient Queue, Auto-conversion of prescription to pharmacy bill.

We have also included standard features like warning of allergies, warning of drug interactions, automatic clinical report generation, automatic referral generation, automatic certificate generation and obstetric calculator. This can store unlimited number of patients, their demographic data, photos, medical records, and visit details. There is a database of clinical diseases including photos and links to resources in Internet which can be expanded by the doctor.

A full featured pharmacy and a laboratory are also included. All the financial details like practice income, expenses are also recorded and analysed.
\end{abstract}

Keywords - Electronic Medical Record system, popularity of software, doctor-computer interaction 